\title{
ON THE EXPONENT OF NORM RESIDUE GROUPS
}

\author{
W. HÜRLIMANN ${ }^{1}$ AND D. SALTMAN
}

\begin{abstract}
We compute the exponent of some norm residue groups in the number theoretic case (global fields). We use the method of Galois cohomology and the theory of the Brauer group over a global field.
\end{abstract}

Let $n$ be a natural number and let $K / k$ be a Galois extension of arbitrary fields with bicyclic group $G=\mathbf{Z} / n \times \mathbf{Z} / n$ generated by $s$ and $t$. By $H^{r}\left(G, K^{*}\right), r \in \mathbf{Z}$, we mean the Tate cohomology groups of $G$ with coefficients in the multiplicative group of $K$. We consider the following diagram of subfields of $K$ and relative norms:

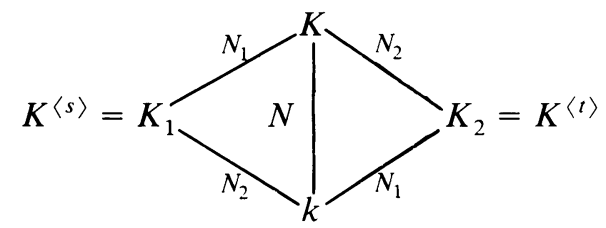

We use the known structure of the third Galois cohomological groups (see [4 and 5]):

$$
H^{3}\left(G, K^{*}\right)=Z^{3} / B^{3} \cong N_{1} K_{2}^{*} \cap N_{2} K_{1}^{*} / N K^{*},
$$

where the 3-cocycles are

$$
Z^{3}=\left\{c=(a, b) \in K_{1}^{*} \times K_{2}^{*} \mid N_{2} a N_{1} b=1\right\}
$$

and the 3-coboundaries are

$$
\begin{array}{r}
B^{3}=\left\{c=(a, b) \in K_{1}^{*} \times K_{2}^{*} \mid \text { there exists }(x, y, z) \in K_{1}^{*} \times K_{2}^{*} \times K^{*}\right. \\
\text { such that } \left.t(x) N_{1} z=a x, s(y)=b y N_{2} z\right\} .
\end{array}
$$

The sets $Z^{3}$ and $B^{3}$ are multiplicative groups through the operation $(a, b)\left(a^{\prime}, b^{\prime}\right)=$ $\left(a a^{\prime}, b b^{\prime}\right)$. The isomorphism in (1) is induced by the map $Z^{3} \rightarrow N_{1} K_{2}^{*} \cap N_{2} K_{1}^{*} / N K^{*}$ which sends $c=(a, b)$ to $N_{2} a=N_{1} b^{-1} \bmod N K^{*}$.

The Diophantine equation $N(x)=a^{n}, a \in k^{*}, x$ an indeterminate with value in $K$, is of interest.

LEMMA. Let $K / k$ be a Galois extension of arbitrary fields with group $G=\mathbf{Z} / n \times$ $\mathbf{Z} / n, n \in \mathbf{N}$. If $H^{3}\left(G, K^{*}\right)=0$, then $k^{*} / N K^{*}$ has exponent at most $n$, that is, every nth power of $k^{*}$ is a norm.

Proof. For all $a \in k^{*}$, the 3-cocycle $c=\left(a, a^{-1}\right)$ is a coboundary. Hence the equation $N(x)=a^{n}$ has a solution.

Received by the editors January 16, 1984.

1980 Mathematics Subject Classification. Primary 12A60; Secondary 12G05, 12E15.

${ }^{1}$ This is a joint work done while the first author was staying at Yale University under a Swiss National Science Foundation fellowship.

1985 American Mathematical Society $0002-9939 / 85 \$ 1.00+\$ .25$ per page 
It is possible to improve on this.

THEOREM 1. Let $K / k$ be a Galois extension of global fields with group $G=Z / p \times$ $Z / p, p$ an odd prime. Then the abelian group $k^{*} / N K^{*}$ is of exponent $p$.

Proof. As $k^{*} / N K^{*}=H^{0}\left(G, K^{*}\right)$ has exponent at most $p^{2}$, it suffices to show that $a^{p}=N(x)$ has a $k$-rational point for every $a \in k^{*}$. The equation $N(x)=a^{p}$ can be written as $N_{1}\left(a N_{2}\left(x^{-1}\right)\right)=1$. Using Hilbert 90 , it suffices to find $d \in K_{2}^{*}$, $x \in K^{*}$ such that

$$
a=N_{2}(x) s(d) d^{-1} .
$$

We interpret this equation in terms of central simple algebras. We introduce the cyclic crossed products $A=\left(K / K_{2}, a\right), B=\left(K_{1} / k, a\right)$ and $C=\left(K / K_{2}, d\right)$. In the Brauer group, we have the following similarity (square brackets denote similarity):

$$
[A]=\left[B \otimes_{k} K_{2}\right] \quad[3,(29.13)] .
$$

W.e write $C^{s}$ for the algebra $C$ with the new $K_{2}$-module structure defined by $k \cdot d=s(k) d, k \in K_{2}, d \in C$. One can show that $C^{s}=\left(K / K_{2}, s(d)\right)$. We observe that solving (2) is the same as finding a central simple $K_{2}$-algebra $C$ such that

$$
[A]=\left[C^{s} \otimes_{K_{2}} C^{\mathrm{op}}\right] .
$$

If $a \in N_{2} K^{*}$, there is nothing to show. Hence we suppose that $A$ is not the trivial algebra. We use the local invariants of Hasse for the description of the Brauer group of a global field [3, Chapter 8 , or 2, Chapter VII]. We will need the exact sequence [3, (32.14)] for the extension $K / K_{2}$. As $A$ is obtained from $B$ by extension of the base field, we have from [2, Theorem 4, p. 113]

$$
(A / w)=n_{v}(B / v) \quad \text { for all places } w \text { of } K_{2} \text { above the place } v \text { of } k \text {. }
$$

Here $(A / w),(B, / w)$ respectively $n_{v}$ denote the local invariants of the classes of $A, B$ respectively the local degree of the extension $\left(K_{2}\right)_{w} / k_{v}$. Thus the computation of the local invariants for the class of $A$ is reduced to a computation concerning the algebra $B$. Let $w$ be a place of $K_{2}$ and $v$ its restriction to $k$. Three cases are possible.

Case 1. If $w$ is an infinite place, it is clear that $(A / w)=0$. Indeed, if $(A / w)=\frac{1}{2}$, then the local index 2 divides the exponent $p$ of the algebra $A$, which is impossible.

Case 2. If $w$ is a finite place invariant under the action of $s$, then $n_{v}=p$ and $(A / w)=(B / v) p$. But $(B / v)=0$ or $s_{v} / p$, with $\left(s_{v}, p\right)=1$, since $[B] \in \operatorname{Br}\left(K_{1} / k\right)$ is of exponent 1 or $p$. It follows that $(A / w)=0$.

Case 3. If the finite place $w$ is not invariant under $s$, we have $p$ places $w=$ $w_{1}, w_{2}, \ldots, w_{p}$ above $v$ and $n_{v}=1$. It follows that $\left(A / w_{i}\right)=(B / v)=0$ or $s_{v} / p$ with $\left(s_{v}, p\right)=1$.

As the next step, we construct a class $[C]$ by giving its local invariants. If $(A / w)=0$, we put $(C / w)=0$. We remark that $s$ is transitive on $w_{1}, w_{2}, \ldots, w_{p}$ and that $\left(C^{s} / w^{s}\right)=(C / w)$. If $\left(A / w_{i}\right)=s_{v} / p$ for $i=1, \ldots, p$, we take the sequence of local invariants

$$
\left\{\left(C / w_{1}\right),\left(C / w_{2}\right), \ldots,\left(C / w_{p}\right)\right\}=\left\{s_{v} / p, 2 s_{v} / p, \ldots,(p-1) s_{v} / p, 0\right\}
$$


such that after appropriate numbering of the $w_{i}$ 's, we have

$$
\left\{\left(C^{s} / w_{1}\right), \ldots,\left(C^{s} / w_{p}\right)\right\}=\left\{2 s_{v} / p, 3 s_{v} / p, \ldots, 0, s_{v} / p\right\} .
$$

As $\sum_{w \mid v}(C / w)=(p-1) s_{v} / 2 \equiv 0 \bmod \mathbf{Z}$ if $p$ is odd, the class [C] is uniquely determined. The field $K$ splits $C$, since if $(C / w) \neq 0$, then $(A / w) \neq 0$, and so $K$ has local degree $p$ at $w$. From the theory of crossed products and the fact that $\operatorname{Br}\left(K / K_{2}\right) \cong H^{2}\left(\langle t\rangle, K^{*}\right) \cong K_{2}^{*} / N K^{*}$, there exists $d \in K_{2}^{*}$ such that $C=$ $\left(K / K_{2}, d\right)$. By construction, the class of $C^{s} \otimes_{K_{2}} C^{\text {op }}$ possesses the same local invariants as the class of $A$. It follows that the algebra $\left(K / K_{2}, a^{-1} s(d) d^{-1}\right)$, which is similar to the algebra $A^{\mathrm{op}} \otimes C^{s} \otimes C^{\mathrm{op}}$, is a matrix algebra. Therefore we have $a^{-1} s(d) d^{-1} \in N_{2} K^{*}$ and the proof is complete.

REMARK. The above proof is not valid if we omit the assumption $p$ is odd. In fact there are biquadratic bicyclic extensions $K / k$ such that $k^{*} / N K^{*}$ is of exponent 4 as we will show below. This illustrates once more the difference in number theory between 2 and the other primes.

TheOREM 2. Let $K / k$ be a Galois extension of global fields with group $G=\mathbf{Z} / 2 \times$ $\mathbf{Z} / 2$. Then we have:

(I) If $H^{3}\left(G, K^{*}\right)=0$, then $k^{*} / N K^{*}$ is of exponent 2 .

(II) If $H^{3}\left(G, K^{*}\right) \neq 0$, then $k^{*} / N K^{*}$ is of exponent 4 .

Proof. In view of the lemma, it remains to show (II). The first author has computed that $H^{3}\left(G, K^{*}\right)=k^{*} / \prod_{i=1}^{3} N_{i} K_{i}^{*}$, where $N_{3}$ is the norm from $K_{3}=K^{\langle s t\rangle}$ to $k$. (Details of proof will appear elsewhere; a connection with the Hasse problem is given below.) On the other side, we have $\Pi_{i=1}^{3} N_{i} K_{i}^{*}=\left\{x \in k^{*} \mid x^{2} \in N K^{*}\right\}[1$, Exercise 5, p. 360]. Since $H^{3}\left(G, K^{*}\right) \neq 0$, there exists $x \in k^{*}$ with $x^{2} \notin N K^{*}$. We are done.

RemarK. One can show that $H^{3}\left(G, K^{*}\right)=N_{1} K_{2}^{*} \cap N_{2} K_{1}^{*} / N K^{*}$ is equal to the group $\{$ local norms $\} /$ global norms $\}$. The question whether a local norm is equal to a global norm is known as the Hasse problem. In the biquadratic case, consider the explicit isomorphism $N_{1} K_{2}^{*} \cap N_{2} K_{1}^{*} / N K^{*} \cong k^{*} / \Pi_{i=1}^{3} N_{i} K_{i}^{*}$, which sends a class $N_{1} a=N_{2} b^{-1} \bmod N K^{*}$ to the class $a N_{K / K_{1}}(d) \bmod \Pi_{i=1}^{3} N_{i} K_{i}^{*}$, where $d$ satisfies $a b=s t(d) d^{-1}$. Using this isomorphism and Exercise 5 in [1], the first author has produced an algorithm which solves the Hasse problem in this particular case (details will appear elsewhere).

\section{REFERENCES}

1. J. W. S. Cassels and A. Fröhlich (Editors), Algebraic number theory, Academic Press, London; Thompson, Washington, D. C., 1967.

2. M. Deuring, Algebren, Springer-Verlag, New York, 1966.

3. I. Reiner, Maximal orders, Academic Press, New York, 1975.

4. S. Takahashi, Cohomology of finite abelian groups, Tôhoku Math. J. (2) 4 (1952), 294-302.

5. T. Tannaka, On the normal form of cohomology groups, J. Math. Soc. Japan 6 (1954), 16-31.

Forschungsinstitut fUr Mathematik der ETH, CH - 8092 ZUrich, SWitzerland

Department of Mathematics, University of Texas, Austin, Texas 78712 